\title{
Splenic peliosis: an unusual cause of splenic lesions
}

\author{
Arshi Yasmin · Pamela McKay $\cdot$ Bob Jackson
}

Received: 1 October 2007 / Accepted: 16 October 2007/Published online: 27 February 2008

(C) The Japanese Society of Hematology 2008

A 49-year-old woman was admitted with a 3-week history of fever, fatigue and night sweats and was found to have abnormal liver function. CT scan showed a normal liver but modestly enlarged spleen, with multiple low attenuation focal lesions (largest $4.1 \mathrm{~cm}$ ). Differential diagnosis included lymphoma, metastases and granulomatous disease. The patient's symptoms resolved spontaneously and liver function tests improved. Repeat CT scan 3 months later was unchanged and she, therefore, underwent splenectomy. A $14.5 \times 10 \times 5.5 \mathrm{~cm}$ spleen was removed containing illdefined haemorrhagic nodules (maximum $3 \mathrm{~cm}$ ). Microscopically, these abnormal areas consisted of dilated bloodfilled spaces with a mixture of histiocytes and lymphocytes. The uninvolved splenic parenchyma had a normal architecture. The overall features were of splenic peliosis.

Peliosis (Greek for blue-black) is a rare disorder of undetermined pathogenesis characterised by blood-filled cystic structures in solid parenchymatous organs, most commonly in the liver but also in the spleen, lungs, bone marrow, kidney and lymph nodes. Splenic peliosis may be idiopathic or associated with infections such as tuberculosis and bartonella, haematological diseases such as myeloma, myelofibrosis, and Hodgkin's disease and with the use of androgenic steroids and erythropoetin. Patients with isolated splenic peliosis may be asymptomatic or describe abdominal discomfort. It is often detected as an incidental finding on abdominal imaging or when

\footnotetext{
A. Yasmin $(\square) \cdot$ P. McKay

Department of Haematology, North Glasgow University

Hospitals NHS Trust, Glasgow, UK

e-mail: arshi.yasmin@northglasgow.scot.nhs.uk

B. Jackson

Department of Pathology, Glasgow Royal Infirmary,

Glasgow, UK
}

spontaneous rupture occurs. In our patient, splenectomy was required to establish a diagnosis but was also therapeutic in view of the potential for spontaneous rupture.
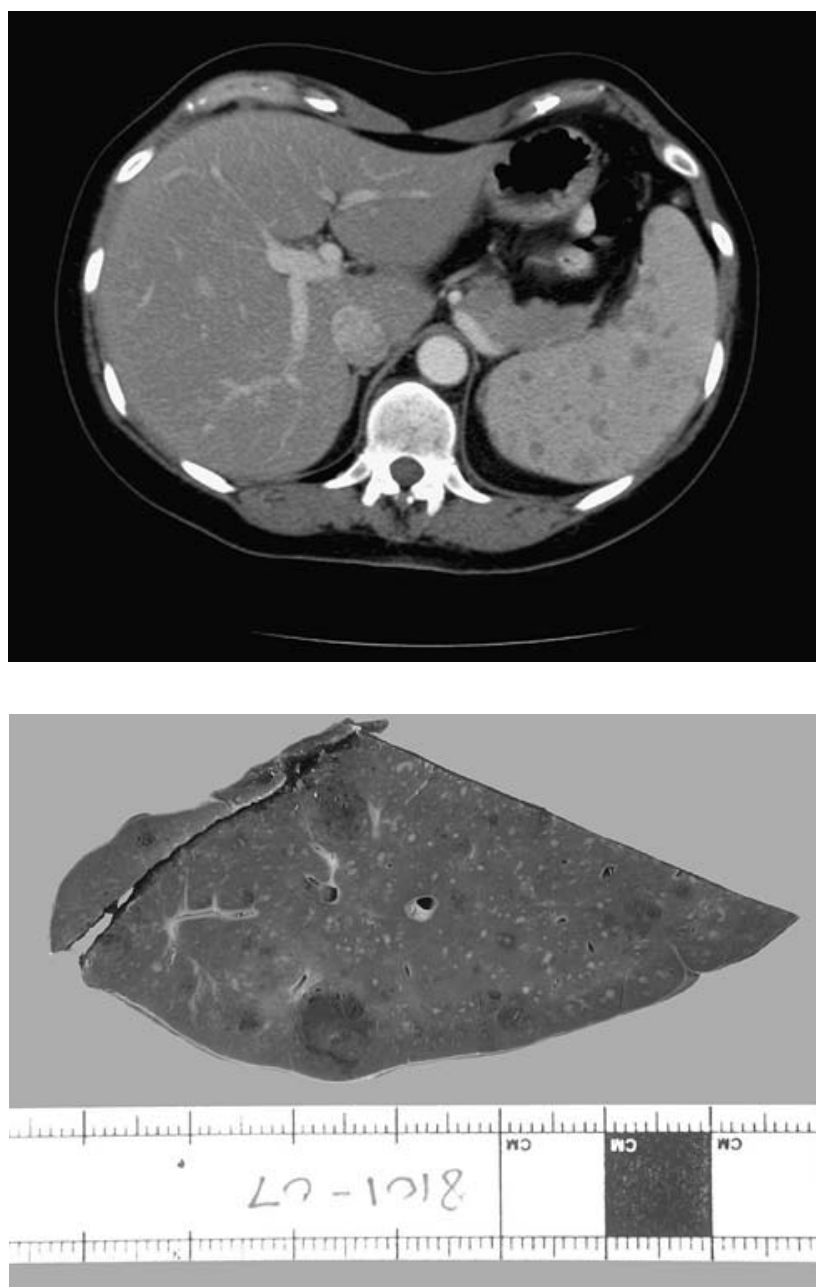